Portland State University

PDXScholar

\title{
Semiconductor Film Grown on a Circular Substrate: Predictive Modeling of Lattice-Misfit Stresses
}

\author{
Ephraim Suhir \\ Portland State University \\ Johann Nicolics \\ Technical University, Vienna \\ G. Khatibi \\ Technical University, Vienna \\ M. Lederer \\ Technical University, Vienna
}

Follow this and additional works at: https://pdxscholar.library.pdx.edu/mengin_fac

Part of the Mechanical Engineering Commons, and the Semiconductor and Optical Materials

Commons

Let us know how access to this document benefits you.

\section{Citation Details}

Suhir, E., Nicolics, J., Khatibi, G., \& Lederer, M. (2016, March). Semiconductor film grown on a circular substrate: predictive modeling of lattice-misfit stresses. In IOP Conference Series: Materials Science and Engineering (Vol. 119, No. 1, p. 012029). IOP Publishing.

This Article is brought to you for free and open access. It has been accepted for inclusion in Mechanical and Materials Engineering Faculty Publications and Presentations by an authorized administrator of PDXScholar. Please contact us if we can make this document more accessible: pdxscholar@pdx.edu. 
International Conference on Materials, Processing and Product Engineering 2015 (MPPE 2015) IOP Publishing IOP Conf. Series: Materials Science and Engineering 119 (2016) 012029 doi:10.1088/1757-899X/119/1/012029

\title{
Semiconductor Film Grown on a Circular Substrate: Predictive Modeling of Lattice-Misfit Stresses
}

\author{
E. Suhir ${ }^{1,2,3}$, J. Nicolics ${ }^{2}$, G. Khatibi ${ }^{2}$, M. Lederer $^{2}$ \\ ${ }^{1}$ Portland State University, Portland, OR, USA; ${ }^{2}$ Technical University, Vienna, \\ Austria, and ${ }^{3}$ ERS Co., Los Altos, CA 94024, USA, Tel. 650-969-1530, cell. 408-410- \\ 0886 \\ suhire@aol.com
}

\begin{abstract}
An effective and physically meaningful analytical predictive model is developed for the evaluation the lattice-misfit stresses (LMS) in a semiconductor film grown on a circular substrate (wafer). The two-dimensional (plane-stress) theory-of-elasticity approximation (TEA) is employed in the analysis. The addressed stresses include the interfacial shearing stress, responsible for the occurrence and growth of dislocations, as well as for possible delaminations and the cohesive strength of a buffering material, if any. Normal radial and circumferential (tangential) stresses acting in the film cross-sections and responsible for its short- and long-term strength (fracture toughness) are also addressed. The analysis is geared to the GaN technology.
\end{abstract}

\section{Introduction}

GaN is a binary III/V direct bandgap semiconductor commonly used in bright light-emitting diodes. GaN based high-electron-mobility-transistor (HEMT) device technology is viewed as a promising one for power amplifier applications [1]. The reliability of GaN devices continues, however, to be a key factor in making promising and viable GaN technology-based devices into reliable products. The elevated lattice-misfit and thermal-mismatch stresses in $\mathrm{GaN}$ films are the major limitations for obtaining high-quality GaN systems on technologically important substrates, such as, e.g., $\mathrm{Si}, \mathrm{SiC}, \mathrm{AlN}$, or diamond (C). A variety of techniques have been suggested to reduce the adverse consequence of the lattice misfit during semiconductor crystal growth (SCG) process. Based on a rather general predictive model for the evaluation of stresses in finite-size bonded joints [2], Luryi and Suhir [3] have shown that the critical thickness of an epitaxial film could be made even infinite, if a properly engineered substrate is used. The idea of employing patterned, porous or otherwise engineered substrates led to many subsequent investigations and to numerous publications exploring the use of nano-sized islands ("towers") as growth nucleation sites. Sagar et al [4] have demonstrated that a reduction in dislocation density from about $10^{10}-10^{12} \mathrm{~cm}^{-2}$ in a template prepared using molecular beam epitaxy (MBE) could be reduced to about $2.5 \times 10^{9} \mathrm{~cm}^{-2}$, if a porous $\mathrm{SiC}$ substrate is employed. The relative level of the latticemisfit and thermal-mismatch stresses in bi-material GaN assemblies was recently addressed, based on analytical (mathematical) predictive modeling, with an objective to evaluate and to compare these two types of stresses [5]. The developed models were based on the strength of material approach (SMA) and treated the $\mathrm{GaN}$ assembly as a bi-material elongated rectangular strip. It was determined that even if a reasonably good lattice match takes place (as, e.g., in the case of a $\mathrm{GaN}$ film fabricated on a $\mathrm{SiC}$ substrate, when the mismatch strain is only about $3 \%$ ) and, in addition, the temperature change (from the fabrication temperature to the operation temperature) was significant (as high as $1000^{\circ} \mathrm{C}$ ), the thermal stresses were still considerably lower than the lattice-misfit stresses. It was determined also that the 
International Conference on Materials, Processing and Product Engineering 2015 (MPPE 2015) IOP Publishing IOP Conf. Series: Materials Science and Engineering 119 (2016) 012029 doi:10.1088/1757-899X/119/1/012029

interfacial shearing and peeling stresses were as important as the normal stresses acting in the crosssections of the $\mathrm{GaN}$ film. While the normal stresses in the $\mathrm{GaN}$ film cross-sections are responsible for the fracture toughness of the film material, it is the interfacial stresses that are responsible for the ability of the assembly to resist delaminations (interfacial cracking) and for the performance of the buffering ("bonding") materials, if any. The objective of the analysis that follows is to develop a simple and physically meaningful predictive TEA based LMS model for a GaN film grown on a circular substrate. Our intent is to evaluate, using the developed model, the applicability and accuracy of the SMA (that addresses a bi-material elongated strip as a more or less suitable substitute for an actual circular assembly).

\section{Analysis}

\subsection{Normal stresses in the assembly mid-portion}

The analysis carried out in this section proceeds from the major assumption that neither the circular configuration of the assembly nor its bow affect the normal LMS in the major mid-portion of a large size bi-material assembly. Let the lattice constants for the materials of the components \#1 (film) and \#2 (substrate) be $a_{1}$ and $a_{2} \leq a_{1}$, respectively, and, as a result of joining these components into a single bi-material assembly, the final interfacial lattice constant is $a$. Then the interfacial strains experienced by the component materials are $\varepsilon_{1}=\frac{a_{1}-a}{a_{1}}=1-\frac{a}{a_{1}}$ in compression and $\varepsilon_{2}=\frac{a-a_{2}}{a_{2}}=\frac{a}{a_{2}}-1$ in tension.

The induced stresses, in accordance with Hooke's law for the two-dimensional state of stress, are

$$
\sigma_{1}=\frac{E_{1}}{1-v_{1}} \varepsilon_{1}=\frac{E_{1}}{1-v_{1}}\left(1-\frac{a}{a_{1}}\right), \sigma_{2}=\frac{E_{2}}{1-v_{2}} \varepsilon_{2}=\frac{E_{2}}{1-v_{2}}\left(\frac{a}{a_{2}}-1\right)
$$

in compression and in tension, respectively. Here $E_{1}$ and $E_{2}$ are Young's moduli, and $v_{1}$ and $v_{2}$ are Poisson's ratios of the materials. The equations (1) reflect the following assumptions: these stresses are the same for all the points in the given cross-section of the given component; the assembly size (in the $\mathrm{x}-\mathrm{y}$ plane) is significant and the assembly points of interest are sufficiently remote from the assembly edges. The corresponding forces are

$$
T_{1}=\sigma_{1} h_{1}=\frac{1}{\lambda_{1}}\left(1-\frac{a}{a_{1}}\right), T_{2}=\sigma_{2} h_{2}=\frac{1}{\lambda_{2}}\left(\frac{a}{a_{2}}-1\right),
$$

where $\lambda_{1}=\frac{1-v_{1}}{E_{1} h_{1}}, \lambda_{2}=\frac{1-v_{2}}{E_{2} h_{2}}$, are the axial compliances of the assembly components, and $h_{1}$ and $h_{2}$ are their thicknesses. The condition $T_{1}=T_{2}$ of equilibrium yields: $a=\frac{\lambda a_{1} a_{2}}{\lambda_{1} a_{1}+\lambda_{2} a_{2}}, \quad$ where $\lambda=\lambda_{1}+\lambda_{2}$. The normal strains in the cross-sections of the assembly components are $\varepsilon_{1}=\lambda_{1} T_{0}, \varepsilon_{2}=\lambda_{2} T_{0}$, where $T_{0}=\frac{a_{1}-a_{2}}{\lambda_{1} a_{1}+\lambda_{2} a_{2}}$ is the longitudinal force (whether $T_{1}$ or $T_{2}$ ). The corresponding normal stresses acting in the cross-sections of the assembly components are $\sigma_{1}=\frac{T_{0}}{h_{1}}=\frac{1}{h_{1}} \frac{a_{1}-a_{2}}{\lambda_{1} a_{1}+\lambda_{2} a_{2}}, \sigma_{2}=\frac{T_{0}}{h_{2}}=\frac{1}{h_{2}} \frac{a_{1}-a_{2}}{\lambda_{1} a_{1}+\lambda_{2} a_{2}}$ in compression and in tension, respectively. When the component \#2 is a thick substrate, and the component \#1 is a thin film, the axial compliance $\lambda_{2}$ of the substrate becomes significantly smaller than the compliance $\lambda_{1}$ of the film, and, if the Young's moduli of the component materials are in the same order of magnitude such as $\mathrm{GaN}$ and $\mathrm{SiC}$, the above formulas can be simplified as follows:

$$
T_{0}=\frac{1}{\lambda_{1}}\left(1-\frac{a_{2}}{a_{1}}\right)=\frac{E_{1} h_{1}}{1-v_{1}}\left(1-\frac{a_{2}}{a_{1}}\right), \sigma_{1}=\frac{E_{1}}{1-v_{1}}\left(1-\frac{a_{2}}{a_{1}}\right),
$$


International Conference on Materials, Processing and Product Engineering 2015 (MPPE 2015) IOP Publishing IOP Conf. Series: Materials Science and Engineering 119 (2016) 012029 doi:10.1088/1757-899X/119/1/012029

These formulas indicate that the normal compressive stress in the mid-portion of the component \#1 (thin film), as long as it is thin enough, is independent of its thickness, and that the normal tensile stress in the substrate is proportional to the thickness ratio and is very low.

\subsection{Interfacial shearing stress}

\subsubsection{Assumptions}

The following major assumptions are used in our analysis:

- The assembly components (the film, and the substrate) can be treated as thin circular plates experiencing small deflections, and the engineering theory of bending of thin plates can be used to predict their physical behavior;

- The peeling stresses do not affect the interfacial shearing stresses and need not be accounted for when evaluating the shearing stresses;

- The interfacial compliances of the assembly in its plane is due to the joint interfacial compliances $\kappa_{1}=\frac{h_{1}}{3 G_{1}}$ and $\kappa_{2}=\frac{h_{2}}{3 G_{2}}$ of the film and the substrate [2];

- The interfacial radial displacements, $u_{1}(r)$, of the component \#1 (film) can be evaluated as the sum of the radial displacements, $u(r)$, caused by the lattice-misfit-induced forces, and additional displacements, $\kappa_{1} \tau_{0}(r)$, of the interfacial point at the given radius $r$, with respect to the displacements $u(r)$ of the inner points of the cross-section: $u_{1}(r)=u(r)+\kappa_{1} \tau_{0}(r) . \quad$ In this formula, $\tau_{0}(r)$ is the interfacial shearing stress in the given cross-section, and $\kappa_{1}$ is the interfacial compliance of the film layer. The displacements $u(r)$ can be evaluated based on the Hooke's law, and are considered the same for all the points of the given (circumferential) cross-section. The second term in this relationship is, in effect, a correction that considers the deviation of the given cross-section from planarity;

- The interfacial radial displacements,. $u_{2}(r)$, of the substrate can be evaluated as $u_{2}(r)=-\kappa_{2} \tau_{0}(r)$.

- Assembly bow has a small effect on the state of stress in the film and need not be accounted for.

- The interfacial shearing stress $\tau_{0}(r)$ increases with an increase in the film thickness and with an increase in the shearing stress gradient $\frac{\partial \tau_{x z}(r)}{\partial z}$ in the through-thickness direction; in an approximate analysis the interfacial shearing stress $\tau_{0}(r)$ can be sought as a product $h_{1} \frac{\partial \tau_{x z}(r)}{\partial z}$ of the film thickness $h_{1}$ and the through-thickness gradient $\frac{\partial \tau_{x z}(r)}{\partial z}$ of the shearing stress at the given radius. This means that the through-thickness gradient $\frac{\partial \tau_{x z}(r)}{\partial z}$ of the shearing stress can be sought in the approximate form: $\frac{\partial \tau_{x z}(r)}{\partial z} \approx \frac{\tau_{0}(r)}{h_{1}}$.

\subsubsection{Basic equation and its solution}

The taken assumption and the condition $u_{1}(r)=u_{2}(r)$ of the displacement compatibility result in the following formula for the radial interfacial displacements of the film:

$$
u(r)=-\kappa \tau_{0}(r),
$$

where $\kappa=\kappa_{1}+\kappa_{2}$ is the total interfacial compliance of the assembly. The formula (4) and the Cauchy formulas [7] $\varepsilon_{r}=u^{\prime}(r), \quad \varepsilon_{\theta}=\frac{u(r)}{r}$ for the normal radial, $\varepsilon_{r}$, and the normal circumferential 
International Conference on Materials, Processing and Product Engineering 2015 (MPPE 2015) IOP Publishing IOP Conf. Series: Materials Science and Engineering 119 (2016) 012029 doi:10.1088/1757-899X/119/1/012029

(tangential), $\varepsilon_{\theta}$, strains yield: $\varepsilon_{r}=-\kappa \tau_{0}^{\prime}(r), \quad \varepsilon_{\theta}=-\kappa \frac{\tau_{0}(r)}{r}$. The corresponding normal stresses in the film can be evaluated, using Hooke's law equations $\sigma_{r}=\frac{E_{1}}{1-v_{1}^{2}}\left(\varepsilon_{r}+v_{1} \varepsilon_{\theta}\right), \quad \sigma_{\theta}=\frac{E_{1}}{1-v_{1}^{2}}\left(\varepsilon_{\theta}+v_{1} \varepsilon_{r}\right)$, as

$$
\sigma_{r}=-\frac{E_{1}}{1-v_{1}^{2}} \kappa\left[\tau_{0}^{\prime}(r)+v_{1} \frac{\tau_{0}(r)}{r}\right], \quad \sigma_{\theta}=-\frac{E_{1}}{1-v_{1}^{2}} \kappa\left[\frac{\tau_{0}(r)}{r}+v_{1} \tau_{0}^{\prime}(r)\right] \text {. }
$$

Introducing the obtained formulas into the equilibrium equation $\frac{\partial \sigma_{r}}{\partial r}+\frac{\partial \tau_{r z}}{\partial z}+\frac{\sigma_{r}-\sigma_{\theta}}{r}=0$, the following basic equation of Bessel type for the shearing stress function, $\tau_{0}(r)$ can be obtained:

$$
\tau_{0}^{\prime \prime}(r)+\frac{\tau_{0}^{\prime}(r)}{r}-\frac{\tau_{0}(r)}{r^{2}}-k^{2} \tau_{0}(r)=0,
$$

where $k=\sqrt{\left(1+v_{1}\right) \frac{\lambda_{1}}{\kappa}}$ is the parameter of the interfacial shearing stress, and $\lambda_{1}$ is the radial compliance of the film. Note that when the SMA is used and the film is significantly thinner than the the substrate, the longitudinal axial compliance $\lambda$ of the assembly is due primarily to the compliance $\lambda_{1}$ of the film, and the parameter of the shearing stress is $k=k_{*}=\sqrt{\frac{\lambda_{1}}{\kappa}}$. The difference should be attributed to the circumferential loading in circular assemblies.

The equation (13) has the following solution:

$$
\tau_{0}(k r)=C_{1} I_{1}(k r) .
$$

where $C_{1}$ is the constant of integration, $k$ is the parameter of the interfacial shearing stress, and $I_{1}(k r)$ is the modified Bessel function of the first kind of the first order [8]. The Bessel function in (7) obeys the following rules of differentiation: $I_{1}^{\prime}(k r)=k I_{0}(k r)-\frac{I_{1}(k r)}{r}, \quad I_{0}^{\prime}(k r)=k I_{1}(k r)$, where $I_{0}(k r)$ is the modified Bessel function of the first kind of zero order. The function $I_{1}(k r)$ is anti-symmetric with respect to the origin and plays the same role as the hyperbolic sine plays in the SMA solution for an elongated strip [2]. Introducing the solution (7) into the formulas (5), we have:

$$
\sigma_{r}=-\frac{E_{1}}{1-v_{1}^{2}} \kappa C_{1}\left[k I_{0}(k r)-\left(1-v_{1}\right) \frac{I_{1}(k r)}{r}\right], \quad \sigma_{\theta}=-\frac{E_{1}}{1-v_{1}^{2}} \kappa C_{1}\left[v_{1} k I_{0}(k r)+\left(1-v_{1}\right) \frac{I_{1}(k r)}{r}\right]
$$

Then the equilibrium equation, with consideration of the the solution (7), yields:

$$
\frac{\partial \sigma_{r}}{\partial r}=C_{1}\left[\frac{E_{1}}{1+v_{1}} \kappa\left(k \frac{I_{0}(k r)}{r}-2 \frac{I_{1}(k r)}{r^{2}}\right)-\frac{I_{1}(k r)}{h_{1}}\right]
$$

This equation can be also written as

$$
\frac{\partial \sigma_{r}}{\partial r}=\frac{C_{1}}{h_{1}}\left[\frac{1-v_{1}}{k^{2}} \frac{d}{d r}\left(\frac{I_{1}(k r)}{r}\right)-\frac{1}{k} \frac{d I_{0}(k r)}{d r}\right] .
$$

By integration, we find

$$
\sigma_{r}=\frac{C_{1}}{h_{1}}\left[\frac{1-v_{1}}{k^{2}} \frac{I_{1}(k r)}{r}-\frac{1}{k} I_{0}(k r)\right]+f(z),
$$

where $f(z)$ is an arbitrary function of the coordinate $z$. Since the radial normal stress in the film does not change in its through-thickness direction, one should put $(z)=C_{0}$, where $C_{0}$ is thus far unknown constant of integration. Thus,

$$
\sigma_{r}=\frac{C_{1}}{h_{1}}\left[\frac{1-v_{1}}{k^{2}} \frac{I_{1}(k r)}{r}-\frac{1}{k} I_{0}(k r)\right]+C_{0} .
$$

There are no external loads acting on the assembly edges, and therefore the boundary condition $\sigma_{r}\left(r_{0}\right)=0$ should be fulfilled. This condition and the expression (12) yield: 
International Conference on Materials, Processing and Product Engineering 2015 (MPPE 2015) IOP Publishing IOP Conf. Series: Materials Science and Engineering 119 (2016) 012029 doi:10.1088/1757-899X/119/1/012029

$$
C_{0}=-\frac{C_{1}}{h_{1}}\left[\frac{1-v_{1}}{k^{2}} \frac{I_{1}\left(k r_{0}\right)}{r_{0}}-\frac{1}{k} I_{0}\left(k r_{0}\right)\right] \text {, }
$$

so that

$$
\sigma_{r}=\frac{C_{1}}{k h_{1}}\left[\frac{1-v_{1}}{k}\left(\frac{I_{1}(k r)}{r}-\frac{I_{1}\left(k r_{0}\right)}{r_{0}}\right)+I_{0}\left(k r_{0}\right)-I_{0}(k r)\right] .
$$

For small radii $r$ the following approximations can be used [8]: $I_{0}(k r) \approx 1, I_{1}(k r) \approx k r$. Then the expression (14) yields:

$$
\sigma_{r}=\frac{C_{1}}{k h_{1}}\left[\left(1-v_{1}\right)\left(1-\frac{I_{1}\left(k r_{0}\right)}{k r_{0}}\right)+I_{0}\left(k r_{0}\right)-1\right] .
$$

Comparing this formula with the first formula in (8), we conclude that the constant $C_{1}$ is expressed as

$$
C_{1}=\frac{1}{2} \frac{E_{1}}{1-v_{1}} \frac{k h_{1}\left(1-\frac{a_{2}}{a_{1}}\right)}{\left(1-v_{1}\right)\left(1-\frac{I_{1}\left(k r_{0}\right)}{k r_{0}}\right)+I_{0}\left(k r_{0}\right)-1} .
$$

This formula indicates particularly that while the radial normal stress is proportional to the $\frac{C_{1}}{k h_{1}}$ ratio and is, hence, film thickness independent, the interfacial shearing stress is proportional to the $C_{1}$ value and increases linearly with an increase in the film thickness.

Using the solution (7) and the formula (16) for the constant of integration $C_{1}$, the following formula for the interfacial shearing stress can be obtained:

$$
\tau_{0}(r)=\tau_{\max } \frac{I_{1}(k r)}{I_{1}\left(k r_{0}\right)},
$$

where $\tau_{\max }=\tau_{0}\left(r_{0}\right)=\frac{E_{1}}{1-v_{1}} k h_{1}\left(1-\frac{a_{2}}{a_{1}}\right) \chi\left(k r_{0}\right)$ is the maximum shearing stress that occurs at the assembly edge, and the function

$$
\chi\left(k r_{0}\right)=\frac{I_{1}\left(k r_{0}\right)}{\left(1-v_{1}\right)\left(1-\frac{I_{1}\left(k r_{0}\right)}{k r_{0}}\right)+I_{0}\left(k r_{0}\right)-1}
$$

considers the effect of the product $k r_{0}$ of the parameter $k$ of the interfacial shearing stress and the assembly size (radius) $r_{0}$ on the maximum interfacial shearing stress. Thus, the maximum interfacial shearing stress at the assembly edge increases with an increase in the effective Young's modulus of the material of the film, with an increase in the parameter $k$ of the interfacial shearing stress and with the increase in the thickness $h_{1}$ of the film. The stress increases, of course, with an increase in the lattice misfit $1-\frac{a_{2}}{a_{1}}$ that plays the role of the "external loading". The maximum shearing stress is inversely proportional to the longitudinal (axial) compliance $\lambda_{1}=\frac{1-v_{1}}{E_{1} h_{1}}$ of the film. The function (18) is tabulated in the second line of Table 1 for $v_{1}=0.25$. As evident from the calculated data, the maximum shearing stress increases with an increase in the parameter $k r_{0}$ when this parameter changes from zero to about $k r_{0} \approx 10.0$, and then remains constant, i.e., assembly size independent.

\subsubsection{Theory-of-elasticity (TEA) vs. strength-of-materials (SMA) solutions}

Let us compare the TEA solution with the SMA solution for the interfacial shearing stress. One can write the SMA solution as [2] 
International Conference on Materials, Processing and Product Engineering 2015 (MPPE 2015) IOP Publishing IOP Conf. Series: Materials Science and Engineering 119 (2016) 012029 doi:10.1088/1757-899X/119/1/012029

$$
\tau(x)=\tau_{\max } \frac{\sinh k x}{\sinh k l},
$$

where the maximum value of the interfacial shearing stress is at the edge of the assembly and is

$$
\tau_{\max }=\tau_{0}(l)=\frac{E_{1}}{1-v_{1}} k h_{1}\left(1-\frac{a_{2}}{a_{1}}\right) \operatorname{coth} \frac{k l}{2} .
$$

Table 1. Tabulated function $\chi\left(\mathrm{kr}_{0}\right)$ that considers the effect of the product $\mathrm{kr}_{0}$ on the maximum interfacial shearing stress and the function $\chi_{1}\left(\mathrm{kr}_{0}\right)$ that considers the effect of this product on the ratio of the maximum interfacial stresses computed based on the theory-of-elasticity approach to the maximum interfacial stress calculated using the strength-of-materials approach

\begin{tabular}{|l|l|l|l|l|l|l|l|l|l|}
\hline$k r_{0}$ & 0 & 0.1 & 0.5 & 1.0 & 2.0 & 3.0 & 5.0 & 10.0 & $\infty$ \\
\hline$\chi$ & 0 & 0.133 & 0.605 & 0.955 & 1.110 & 1.085 & 1.043 & 1.021 & 1.000 \\
\hline$\chi_{1}$ & 0 & 0.00665 & 0.1512 & 0.4622 & 0.9823 & 1.2972 & 1.5152 & 1.6085 & 1.5811 \\
\hline
\end{tabular}

Comparing the TEA solution with the SMA solution we conclude that the distribution of the interfacial shearing stress along the assembly radius (length) is governed, in the case of the TEA solution, by the ratio $\frac{I_{1}(k r)}{I_{1}\left(k r_{0}\right)}$ of the modified Bessel functions, and by the ratio $\frac{\sinh k x}{\sinh k l}$ of the hyperbolic sines, in the case of the SMA solution. Another, more important, difference is due to the considerably higher value of the parameter $k$ of the interfacial shearing stress in the TEA solution. The maximum TEA shearing stress $\tau_{\max }^{T E A}$ can be determined from the maximum SMA shearing stress $\tau_{\max }^{S M A}$ as

$$
\tau_{\max }^{T E A}=\frac{k_{T E A}}{k_{S M A}} \chi\left(k_{T E A} r_{0}\right) \tanh \left(\frac{k_{S M A} l}{2}\right) \tau_{\max }^{S M A}=\sqrt{\left(1+v_{1}\right)} \chi\left(k r_{0}\right) \tanh \left(\frac{k r_{0}}{2 \sqrt{2\left(1+v_{1}\right)}}\right) \tau_{\max }^{S M A}=\chi_{1}\left(k r_{0}\right) \tau_{\max }^{S M A}
$$

where the function $\chi_{1}\left(k r_{0}\right)=\sqrt{\left(1+v_{1}\right)} \chi\left(k r_{0}\right) \tanh \left(\frac{k r_{0}}{2 \sqrt{\left(1+v_{1}\right)}}\right)$ is the maximum interfacial stress ratio.

The half-assembly-length $l$ is replaced here with the radius $r_{0}$ value, and the notation $k=k_{T E A}$ is used. The function $\chi\left(k r_{0}\right)$ changes from zero to one, when the product $k r_{0}$ changes from zero to infinity. For small $k r_{0}$ values this function can be computed as $\chi\left(k r_{0}\right)=\frac{k r_{0}}{1-v_{1}}$. The function $\chi_{1}\left(k r_{0}\right)$ changes from $\chi_{1, \text { min }}=k r_{0} \chi_{\min }=\frac{\left(k r_{0}\right)^{2}}{1-v_{1}}$ to $\chi_{1 \text { max }}=\sqrt{1+v_{1}} \chi_{\text {max }}$, when the product $k r_{0}$ changes from zero to infinity (actually, from a small number smaller than, say, 2, to a large number exceeding 10). The calculated values of the function $\chi_{1}\left(k r_{0}\right)$ are shown in the bottom line of Table 1 . The computed data indicate that the SMA underestimates the maximum shearing stress that takes place for large $k r_{0}$ values $\left(k r_{0} \succ 3\right)$ and overestimates this stress for small $k r_{0}$ values $\left(k r_{0} \prec 2\right)$. This is due primarily to the different values of the parameter $k$ of the interfacial stress: this parameter is by the factor of $\sqrt{1+v_{1}}$, greater in the case of the TEA based solution. If this parameter were the same in the two approaches, then the shearing stress predicted for large enough $\left(k r_{0} \succ 10\right)$ assemblies on the basis of the TEA and SMA would be exactly the same. This means that, in an approximate stress analysis of large size and/or stiff assemblies $\left(k r_{0} \succ 10\right)$, one can compute the parameter $k$ using the TEA formula and then calculate the maximum interfacial shearing stress on the basis of the simpler SMA formula. The functions $\frac{I_{1}(k r)}{I_{1}\left(k r_{0}\right)}$ and $\frac{\sinh (k r)}{\sinh \left(k r_{0}\right)}$ reflect the distributions of the interfacial stress along the assembly in the cases of TEA and SMA based solutions, respectively. The parameter $k$ of the interfacial shearing stress is assumed to be the same in 
International Conference on Materials, Processing and Product Engineering 2015 (MPPE 2015) IOP Publishing IOP Conf. Series: Materials Science and Engineering 119 (2016) 012029 doi:10.1088/1757-899X/119/1/012029

the TEA and in the SMA based solutions. This means that TEA predicts somewhat higher stress concentration at the assembly ends than the SMA (for the same maximum stress at the assembly end). The predicted relative ordinates of the interfacial shearing stress are rather close though.

For large arguments $z(z \succ 10)$, the modified Bessel function of the order $n$ can be evaluated by the approximate formula [8]: $I_{n}(z)=\frac{e^{z}}{\sqrt{2 \pi z}}$. Then, with

$$
I_{0}\left(k r_{0}\right) \cong I_{1}\left(k r_{0}\right) \cong \frac{e^{k r_{0}}}{\sqrt{2 \pi k r_{0}}}, \quad I_{0}(k r) \cong I_{1}(k r) \cong \frac{e^{k r}}{\sqrt{2 \pi k r}}
$$

we obtain:

$$
\tau_{0}(r)=\tau_{\max } \sqrt{\frac{r_{0}}{r}} e^{-k\left(r_{0}-r\right)}
$$

where the maximum value of the shearing stress may be derived from the formulas (20) and (21). As evident from the formula (23), the interfacial shearing stress, $\tau_{0}(r)$, concentrates along a narrow peripheral ring, and is next to zero for the inner radii of the assembly $r \prec \prec r_{0}$.

\subsection{Normal stresses}

Introducing the expression (16) for the constant $C_{1}$ into the formula (15) for the normal stress $\sigma_{r}(r)$ in the film, we obtain:

$$
\sigma_{r}=\sigma_{1}\left[1-\frac{\left(1-v_{1}\right)\left(1-\frac{I_{1}(k r)}{k r}\right)+I_{0}(k r)-1}{\left(1-v_{1}\right)\left(1-\frac{I_{1}\left(k r_{0}\right)}{k r_{0}}\right)+I_{0}\left(k r_{0}\right)-1}\right]
$$

where $\sigma_{1}$ is the normal stress in the mid-portion of the assembly. The expression in the brackets is, in effect, a "correction" that considers the role of the finite radius $r_{0}$ of the assembly. By differentiation we find:

$$
\frac{d \sigma_{r}}{d r}=-\sigma_{1}\left[\frac{\frac{1-v_{1}}{k r}\left(2 \frac{I_{1}(k r)}{r}-k I_{0}(k r)\right)+k I_{1}(k r)}{\left(1-v_{1}\right)\left(1-\frac{I_{1}\left(k r_{0}\right)}{k r_{0}}\right)+I_{0}\left(k r_{0}\right)-1}\right]
$$

Then the circumferential stress $\sigma_{\theta}$ can be found from the equilibrium equation as follows:

$$
\sigma_{\theta}=\sigma_{r}+r \frac{d \sigma_{r}}{d r}+2 \frac{\tau_{0}(r)}{h_{1}} r=\sigma_{1}\left[1+\left(1-v_{1}\right) \frac{I_{0}(k r)-\frac{I_{1}(k r)}{k r}}{\left(1-v_{1}\right)\left(1-\frac{I_{1}\left(k r_{0}\right)}{k r_{0}}\right)+I_{0}\left(k r_{0}\right)-1}\right]
$$

This stress changes from $\sigma_{1}$ in the midportion of the assembly, where it is not different from the normal radial stress, to the

$$
\sigma_{\theta}\left(r_{0}\right)=\sigma_{1} \frac{\left(1-v_{1}\right)\left(I_{0}\left(k r_{0}\right)-2 \frac{I_{1}\left(k r_{0}\right)}{k r_{0}}\right)}{\left(1-v_{1}\right)\left(1-\frac{I_{1}\left(k r_{0}\right)}{k r_{0}}\right)+I_{0}\left(k r_{0}\right)-1}
$$

value at the assembly end. Clearly, this stress cannot be found on the SMA basis. 
International Conference on Materials, Processing and Product Engineering 2015 (MPPE 2015) IOP Publishing IOP Conf. Series: Materials Science and Engineering 119 (2016) 012029 doi:10.1088/1757-899X/119/1/012029

In the case of a large size (large $r_{0}$ values) and/or stiff (large $k$ values) assemblies, the formulas for the normal stresses can be simplified:

$$
\sigma_{r}=\sigma_{1}\left[1-\sqrt{\frac{r_{0}}{r}} e^{-k\left(r_{0}-r\right)}\right], \quad \sigma_{\theta}=\sigma_{1}\left[1+\left(1-v_{1}\right) \sqrt{\frac{r_{0}}{r}} e^{-k\left(r_{0}-r\right)}\right] .
$$

These formulas indicate that the normal stresses, $\sigma_{r}$ and $\sigma_{\theta}$, in the film are uniformly distributed over the inner portion of the assembly $\left(r \prec \prec r_{0}\right)$. At the assembly end the radial stress $\sigma_{r}$ is zero, and the circumferential stress is

$$
\sigma_{\theta}=\left(2-v_{1}\right) \sigma_{1}
$$

i.e., by the factor of $2-v_{1}$ higher than the normal stresses, $\sigma_{1}$, in the mid-portion of the film.

\section{Numerical example}

\subsection{Input Data}

\begin{tabular}{|l|l|l|}
\hline Component & $\begin{array}{l}\text { Film, GaN } \\
(\text { Component \#1) }\end{array}$ & $\begin{array}{l}\text { Substrate,SiC } \\
\text { (Component \#2) }\end{array}$ \\
\hline Young's modulus & $181000 \mathrm{MPa}$ & $461070 \mathrm{MPa}$ \\
\hline Poisson's ratio & 0.352 & 0.175 \\
\hline Lattice constant & $3.18 \mathrm{~A}$ & $3.08 \mathrm{~A}$ \\
\hline Thickness & $0.002 \mathrm{~mm}$ & $0.100 \mathrm{~mm}$ \\
\hline Assembly radius $r_{0}=25.4 \mathrm{~mm}$ & \\
\hline
\end{tabular}

\subsection{Calculated plots}

The calculated stresses for the example of $2 \mu \mathrm{m} \mathrm{GaN} \mathrm{film} \mathrm{on} \mathrm{a} \mathrm{SiC} \mathrm{substrate} \mathrm{are} \mathrm{presented} \mathrm{in} \mathrm{figures} 1$ and 2. Figure 1 displays plots of interfacial shearing stress calculated by using TEA and SMA, respectively and the plots of radial and tangential stresses based on TEA are shown in figure 2. Experimental studies show that thermally induced stresses in epitaxial grown GaN layers may relax by cracking or occurrence of high dislocation densities during the cooling down from deposition temperatures. Still biaxial stresses with values exceeding $1 \mathrm{GPa}$ have been observed as a consequence of lattice-misfit and thermal-mismatch strains on $\mathrm{GaN}$ epitaxial films deposited on $\mathrm{SiC}$ substrates $[4,9]$.

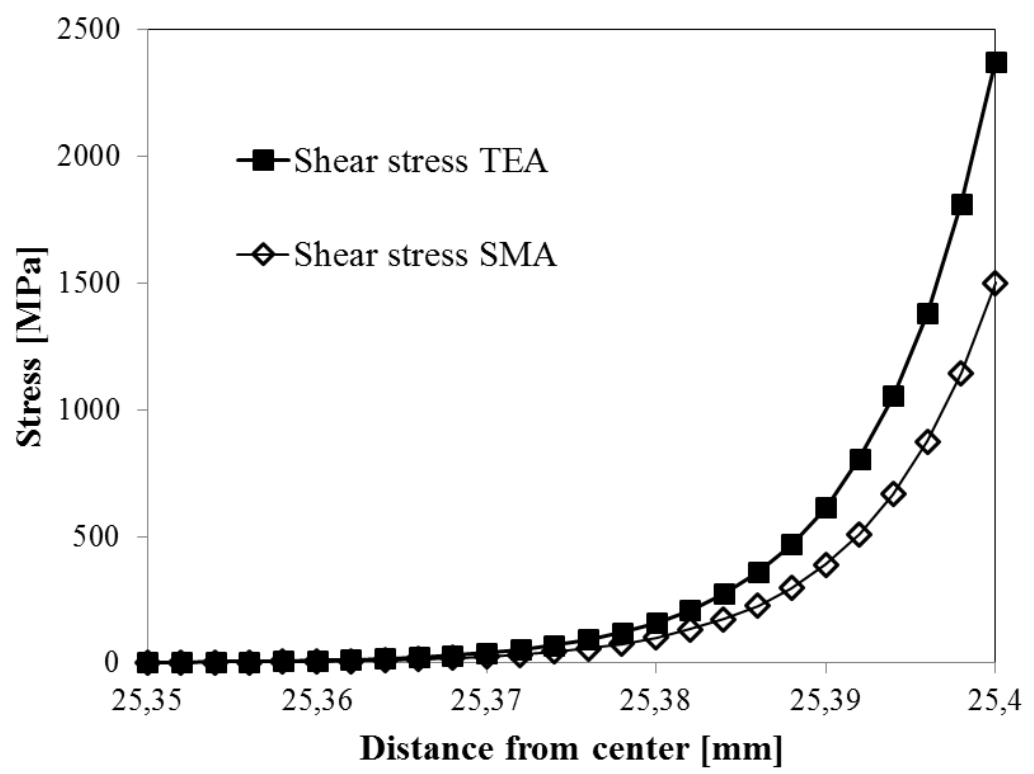

Figure 1. Plots of interfacial shearing stress at the assembly end. 


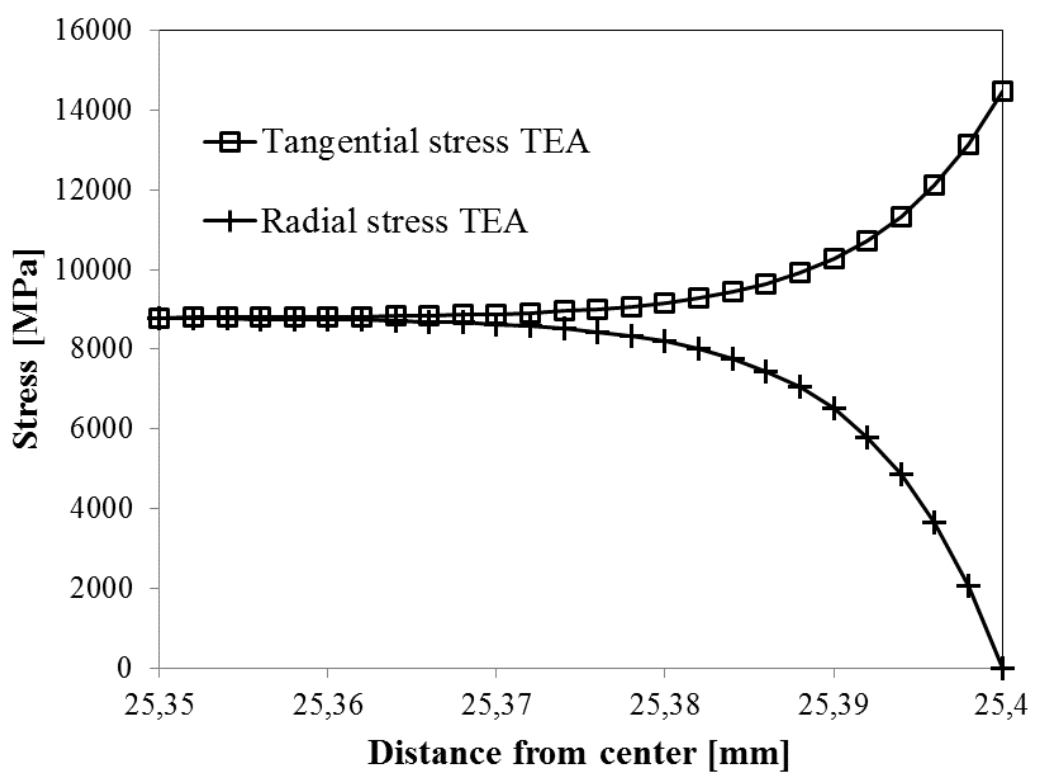

Figure 2. Plots of tangential and radial stresses at the assembly end.

\section{Conclusions}

- The obtained theory-of-elasticity solution enables one to estimate the state of stress normal in a $\mathrm{GaN}$ film.

- The computed peripheral shearing stresses and circumferential stresses are significant. It is envisioned that the occurrence of dislocations in thin semiconductor films starts at their peripheral portions and then rapidly propagates inwards the assembly, until the relief in the shearing stresses in the inner portion of the assembly is sufficient to "arrest" the further penetration of dislocations.

- The theory-of-elasticity solution predicts that the state of stress in the assembly in question becomes assembly size independent, when the product $k r_{0}=\sqrt{3 \frac{1-v_{1}^{2}}{1+v_{2}} \frac{E_{2}}{E_{1}}} \frac{r_{0}}{\sqrt{h_{1} h_{2}}}$ of the parameter $k$ of the interfacial shearing stress and the assembly radius $r_{0}$ exceeds 10 . This takes place when the modified Bessel functions of the first kind of the first and the second order become sufficiently large and equal to each other at a sufficiently high current radius $r$ value. In the above formula, $E_{1}$ and $E_{2}$ are Young's moduli of the film and the substrate materials, $v_{1}$ and $v_{2}$ are their Poisson's ratios, $h_{1}$ and $h_{2}$ are the thicknesses of the film and the substrate, and $r_{0}$ is the assembly radius.

- The theory-of-elasticity and the strength-of-materials predictions lead to the same $k r_{0}$ criterion if the parameter $k$ of the interfacial shearing stress is evaluated based on the theory-of-elasticity formula, which predicts the parameter $k$ value that is by the factor of $\sqrt{1+v_{1}}$ larger than the strength-of-material prediction.

- The following simple formulas can be used to evaluate the maximum elastic stresses in a bi-material circular semiconductor thin film large size assembly:

$$
\sigma_{r, \max }=\sigma_{1}=\frac{E_{1}}{1-v_{1}} \varepsilon, \quad \varepsilon=1-\frac{a_{2}}{a_{1}}, \quad \tau_{\max }=k h_{1} \sigma_{1}, \sigma_{\theta, \max }=\left(2-v_{1}\right) \sigma_{1} .
$$

Here $\sigma_{1}$ is the maximum normal radial and normal circumferential (tangential) stress in the film's midportion, $\varepsilon$ is the lattice-misfit strain, $a_{1}$ and $a_{2}$ are the lattice constants of the substrate and the film materials (the film is in compression if the strain $\varepsilon$ is positive), $\tau_{\max }$ is the maximum interfacial shearing stress at the assembly end, $k$ is the parameter of the interfacial shearing stress, $h_{1}$ is the film thickness, and $\sigma_{\theta, \max }$ is the maximum normal circumferential stress at the assembly end. 
International Conference on Materials, Processing and Product Engineering 2015 (MPPE 2015) IOP Publishing IOP Conf. Series: Materials Science and Engineering 119 (2016) 012029 doi:10.1088/1757-899X/119/1/012029

- The developed predictive model can be used, in addition to the routine finite-element analyses (FEA), for the assessment of the merits and shortcomings of a particular semiconductor-crystal growth technology, as far as the expected lattice-misfit stresses are concerned, before the actual experimentation and/or fabrication is considered and conducted. The models can be used particularly to determine, from the observed critical film thickness, the material, design and technological factors that lead to elevated dislocation densities.

\section{Acknowledgement}

M. Lederer and G. Khatibi wish to thank Christian Doppler Gesellschaft, Vienna Austria (CD labor RELAB).

\section{References}

[1] Lee J et al, Wafer bonding of SiC and GaN, MRS Symp. Proc., vol. 681E, MRS, 2001

[2] Suhir E, Stresses in bi-metal thermostats, ASME J. of Appl. Mech., vol.55, No.4, 1986

[3] Luryi S, Suhir E, New approach to the high quality epitaxial growth of lattice-mismatched materials, Appl. Phys. Lett., vol.49, No. 140, 1986

[4] Sagar A, Feenstra R M, Growth of GaN on porous SiC by molecular beam epitaxy in Porous Silicon Carbide and Gallium Nitride: Epitaxy, Catalysis, and Biotechnology Applications, ed Feenstra R M, Wood C E C, Wiley, West Sussex, 2008

[5] Suhir E, Stresses in Bi-Material GaN Assemblies, J. Appl. Phys. 110, 074506, 2011

[6] Suhir E, Predicted Stresses in a Circular Substrate/Thin-Film System Subjected to the Change in Temperature, Journal of Applied Physics, vol.88, No.5, 2000

[7] Timoshenko S, and Goodier, J N, Theory of Elasticity, $3^{\text {rd }}$ ed., McGraw-Hill, New York, 1970

[8] Sneddon I N, Special Functions of Mathematical Physics and Chemistry, Oliver and Boyd, Edinburgh, 1956

[9] Ahmad I, Holtz M, Faleev N N, Temkin H, Dependence of the stress-temperature coefficient on dislocation density in epitaxial GaN grown on $\alpha-\mathrm{Al}_{2} \mathrm{O}_{3}$ and $6 \mathrm{H}-\mathrm{SiC}$ substrates, J. Appl. Phys. vol.95, No.4, 2004 